\title{
Association of high sensitive C-reactive protein with coronary heart disease: a Mendelian randomization study
}

\author{
Qian Zhuang ${ }^{1}$, Chong Shen², Yanchun Chen ${ }^{1}$, Xianghai Zhao ${ }^{1}$, Pengfei Wei ${ }^{1}$, Junxiang Sun ${ }^{1}$, Yanni Ji',
} Xiaotian Chen ${ }^{3}$ and Song Yang ${ }^{1 *}$ (1)

\begin{abstract}
Objectives: Whether high sensitivity C-reactive protein (hs-CRP) has a causal effect on coronary heart disease (CHD) is unclear. This study investigated the causal effect of hs-CRP on CHD risk using Mendelian Randomization (MR) analysis.

Methods: A total of 3802 subjects were recruited in the follow-up study. Linear regression model was used to evaluate the relationship between CRP polymorphisms and hs-CRP. Survival receiver operator characteristic curve method was used to explore the cut-off of hs-CRP on CHD incidence. Cox regression model was applied to detect the association of hs-CRP with CHD by calculating the hazard ratio (HR) and 95\% confidence interval (CI). Rs1205 and rs876537 in CRP were selected as instrumental variables in MR analysis.

Results: During a median follow-up time of 5.01 years, 98 CHD incidence was identified (47.03/104 person-years). Hs-CRP was significantly increased among rs 1205 and rs876537 genotypes with $r$ values of 0.064 and 0.066 , respectively. Hs-CRP $1.08 \mathrm{mg} / \mathrm{L}$ was identified as the cut-off value with a maximum value of sensitivity and specificity on prediction of CHD. Participants with $\geq 1.08 \mathrm{mg} / \mathrm{L}$ of hs-CRP has a higher risk of CHD incidence than that of participants with $<1.08 \mathrm{mg} / \mathrm{L}$, the adjusted $H R(95 \% C()$ was $1.69(1.11-2.60)$ with a $P$ value of 0.016 . No significant casual association was observed between hs-CRP and CHD with a $P$ value of 0.777 .
\end{abstract}

Conclusions: The association between hs-CRP and CHD is unlikely to be causal, hs-CRP might be a predictor for incidence of CHD in general population.

Keywords: Hs-CRP, CRP gene, CHD, Mendelian randomization analysis

\section{Background}

Coronary heart disease (CHD) is one of the leading global cause of death and disability. The current situation of rapid aging in China makes CHD become the 4th leading cause of mortality [1]. The increased adiposity, type 2 diabetes mellitus (T2DM), hypertension, sedentary lifestyle, as well as genetic factors, are the well-known determinants of CHD [2].

Inflammation promotes endothelial cell damage and atherogenesis, is an important risk factor for CHD in clinical [3]. As a famous parameter of inflammation, C-

\footnotetext{
* Correspondence: 13921382937@163.com

${ }^{1}$ Department of Cardiology, Affiliated Yixing People's Hospital of Jiangsu University, Yixing, China

Full list of author information is available at the end of the article
}

reactive protein (CRP) is also considered a valuable predictor of CHD risk [4]. To meet the super-precision of clinical practice, the measurement of high sensitivity $\mathrm{C}$ reactive protein (hs-CRP) is proposed for its better capacity in identifying the individuals at high risk for CHD [5]. Hs-CRP has commonly been used for cardiovascular diseases (CVDs) risk stratification. The elevated level of serum hs-CRP was significantly increased the risk of myocardial infarction [6]. Randomized clinical trials have observed that the use of statins in individuals with elevated hs-CRP was associated with a reduction in hs-CRP and a decreased risk of vascular events [7]. Even numerous of studies support that the hs-CRP is an established marker for future risk of CVDs, however, inconsistent findings have been reported on CHD $[8,9]$. Therefore, it

(c) The Author(s). 2019 Open Access This article is distributed under the terms of the Creative Commons Attribution 4.0 International License (http://creativecommons.org/licenses/by/4.0/), which permits unrestricted use, distribution, and reproduction in any medium, provided you give appropriate credit to the original author(s) and the source, provide a link to the Creative Commons license, and indicate if changes were made. The Creative Commons Public Domain Dedication waiver (http://creativecommons.org/publicdomain/zero/1.0/) applies to the data made available in this article, unless otherwise stated. 
has considerable interest in establishing whether hs-CRP has a causal role in CHD.

Mendelian randomization (MR) studies utilize genetic variants (such as he-CRP) as instrumental variables (IVs) to investigate possible causal relationship between exposure and outcomes through an intermediate trait [10]. The dominant advantage of MR studies is that they are not as vulnerable to confounding and reverse causality [11, 12]. If the intermediate trait is causally linked to disease, then genetic variants influencing the trait should also influence disease risk. Indeed, MR has been regarded as nature's analogue of randomized controlled trials, thus, it has also been used in cardiovascular research to explore novel potential etiologic mechanisms and enhance our understanding of current therapies $[13,14]$.

The aim of the current study was to identify common single nucleotide polymorphisms (SNPs) in CRP that influence hs-CRP levels, and use the concept of MR to improve understanding of the possible causal relationship of hs-CRP levels with CHD.

\section{Methods}

\section{Study population}

A total of 4222 participants were drawn from the community hypertension survey in Yixing city, China, 2009 [15]. After we excluded the individuals with a history of CHD $(n=50)$, elder participants $(n=94)$ and missing measurement of hs-CRP $(n=373), 3802$ subjects were finally enrolled in the follow-up study.

To access the incidence of disease status in the followup study, both concentrated and household surveys were conducted. Data collected from the local hospitals, Centers for Disease Control and Prevention, community health service centers and social security center was further inspected to reduce the information bias. During a median follow-up time of 5.01 years, 98 new-onset CHDs were recorded.

All participants were interviewed and underwent physical examinations and laboratory tests. The demographic characteristics of the participants were obtained by trained research staff though a standard questionnaire. The weight, height and 3 blood pressure measurements were obtained from each participant by trained and certified observers according to a standard protocol.

Written informed consent was obtained from all participants. The research protocol was approved by the ethics committee of Nanjing Medical University and written informed consent was obtained from all subjects during epidemiological interviews.

\section{SNP selection}

The CRP gene was located on chromosome 1q23.2 (Gene ID: 1401; NC_000001.11) and spans $2.3 \mathrm{kbps}$ and contains 4 exons. We searched the SNPs covered CRP gene from the upstream $5 \mathrm{~kb}$ to the downstream $2 \mathrm{~kb}$ and selected tagging SNPs (tagSNPs) from the database of the Chinese Han population in Beijing, China of the International Hap MAP Project (HapMap Data Rel 24/ phase II Nov08, on NCBI B36 assembly, dbSNPb126). All tagSNPs were selected with a minor allele frequency $(\mathrm{MAF}) \geq 0.05$ and linkage disequilibrium (LD) $r^{2} \geq 0.8$. We also applied a functional candidate strategy to select potential functional SNPs on the bioinformatics effect prediction website (SNPINFO, https://snpinfo. niehs.nih.gov/). Finally, rs1205(C> T), rs1073715(C > $\mathrm{T}), \operatorname{rs} 876537(\mathrm{C}>\mathrm{T})$ and $\operatorname{rs} 2808630(\mathrm{C}>\mathrm{T})$ were selected. The specific biological information was summarized in the Additional file 1: Table S1.

\section{Blood sampling}

Blood samples were taken from participants after an over-night fasting $(>10 \mathrm{~h}$ ), adding ethylenediamine tetraacetic acid (EDTA)-containing receptacles. The blood samples were centrifuged at $3500 \mathrm{rpm}$ to separate the serum and stored at $-20^{\circ} \mathrm{C}$. Serum hs-CRP, total cholesterol (TC), triglycerides (TG), high-density lipoprotein cholesterol (HDL-C), low-density lipoprotein cholesterol (LDL-C) and glucose (GLU) were measured by an automatic biochemical analysis system of Siemens (Advia 1200, German).

Following recommendations from the American Centers for Disease Control and the American Heart Association [16], the participants with hs-CRP $\geq 1.0 \mathrm{mg} /$ $\mathrm{L}$ and hs-CRP $<1.0 \mathrm{mg} / \mathrm{L}$ correspond to high risk group (HRG) and normal group (NG), respectively.

\section{SNP genotyping}

DNA was extracted using a standard phenole-chloroform method. Genotyping was performed using the TaqMan allelic discrimination assay in 384-well plates on the platform of $7900 \mathrm{HT}$ Real-time polymerase chain reaction (PCR) System (Applied Biosystems, Foster City, CA). The primers and probes were designed using Primer Express Oligo Design software v2.0 (ABI PRISM). Genotyping results were determined using SDS 2.3 Allelic Discrimination Software (Applied Biosystems). Meanwhile, each plate was included blank samples as negative controls for the genotyping quality confirmation. The successful call rates of SNPs rs10737175, rs1205, rs2808630 and rs2966449 were 98.44, 98.31, 98.39 and $98.41 \%$, respectively.

\section{Statistical analysis}

Unpaired Student's t-test was used to test the differences in of all the quantitative variables among groups presented as means \pm standard deviation (SD). Chi-square $\left(\chi^{2}\right)$ test was performed to compare the proportion of hypertension, T2DM, smoking and drinking between 
HRG and NG group. Linear regression model was used to analyze the correlation between the variations of $C R P$ gene and hs-CRP. Besides, we used survival receiver operator characteristic curve (SROC) to explore the cut-off values of hs-CRP with CHD incidence. Cox proportional hazard regression model was applied to evaluate the association of $C R P$ gene with $C H D$ by calculating hazard ratio $(H R)$ and $95 \%$ confidence interval $(C I)$.

The MR analysis for exploring the causal effect of hsCRP on the risk of CHD applying inverse-variance weighted (IVW) methods, where the SNPs are deemed as IVs. The basic principle of MR analysis for the current study was shown in Fig. 1. Effects are given as odds ratios $(O R)$ and $95 \% C I$. A two-tailed $P$ value of 0.05 was defined as the cut-off for statistical significance. The SROC and MR analysis were undertaken using the $R$ Packages of "survivalROC" and "Mendelian Randomization".

\section{Results}

\section{Demographic characteristics at baseline}

The demographic characteristics of participants were summarized in Table 1. The age, BMI, SBP, DBP, TC, TG, LDL-C, GLU and proportion of hypertension in HRS were significantly higher than NG, whereas HDL$\mathrm{C}$, the proportion of T2DM, smoking and drinking were significantly lower $(P<0.05)$. Overall, these characteristics were adjusted as confounding factors in the multivariate regression analysis.

\section{Association of CRP polymorphisms with CHD incidence}

During a median follow-up time of 5.01 years, $98 \mathrm{CHD}$ incidence was identified with an incidence density of 47.03/ $10^{4}$ person-years. No significant associations were observed between each of the four SNPs with CHD in the whole population. After adjusted for age, gender, BMI, HDL-C, LDL-C, TC, TG, smoking, drinking, hypertension and T2DM, the results were still not significant (Table 2). Stratification analysis by age ( 55 years), gender, smoking and drinking status were further conducted, however, no significant associations were found between $C R P$ and CHD (Additional file 1: Table S2).

\section{Association of hs-CRP with CHD}

The incidence density of CHD for HRG and NG group were $71.08 / 10^{4}$ and $33.79 / 10^{4}$ person-years, respectively. The elevated hs-CRP was significantly increased CHD risk, the $H R(95 \% C I)$ was $2.05(1.36-3.09)$ with a $P$ value of 0.001 . But, the result was still not significant after adjusted for cofounders, the adjusted $H R(95 \% C I)$ was 1.54 (0.99-2.36) with a $P$ value of 0.051 .

The SROC analysis showed that the highest sensitivity and specificity of cut-off point for hs-CRP to predict CHD incidence was $1.08 \mathrm{mg} / \mathrm{L}$, the sensitivity and specificity were 60.2 and $59.1 \%$, respectively (Additional file 1 : Figure S1). Participants with $\geq 1.08 \mathrm{mg} / \mathrm{L}$ of hs-CRP has a higher risk of $\mathrm{CHD}$ incidence than that of participants with $<1.08 \mathrm{mg} / \mathrm{L}\left(74.92 / 10^{4}\right.$ vs $32.97 / 10^{4}$ person-years), the adjusted $H R(95 \% C I)$ was $1.69(1.11-2.60)$ with a $P$ value of 0.016 . Detailed results were displayed in Table 3.

\section{Correlation of CRP variation with hs-CRP}

$C R P$ rs1205 and rs876537 $\mathrm{C}>\mathrm{T}$ variations were significantly correlated with hs-CRP, after adjusted for age, gender, BMI, TC, TG, HDL-C, LDL-C, smoking, drinking, T2DM and hypertension, the standard regression coefficients were 0.064 and 0.066 , respectively, $P<0.001$. No significant correlations were observed between rs10737175, rs2808630 and hs-CRP, with $P$ values of 0.713 and 0.271 (Additional file 1: Table S3). Thus, we selected rs1205 and rs876537 were selected as IVs in the MR analysis.

\section{MR analysis between hs-CRP and CHD}

The causal effect was evaluated by using MR analysis assessing the association of genetically predicted hs-CRP with CHD risk (Table 4). The crude model showed that hs-CRP was not statistically associated with $\mathrm{CHD}$, the cruder $O R(95 \% C I)$ was $1.04(0.67-1.62)$ with a $P$ value of 0.855 . The result remained negative after adjustment

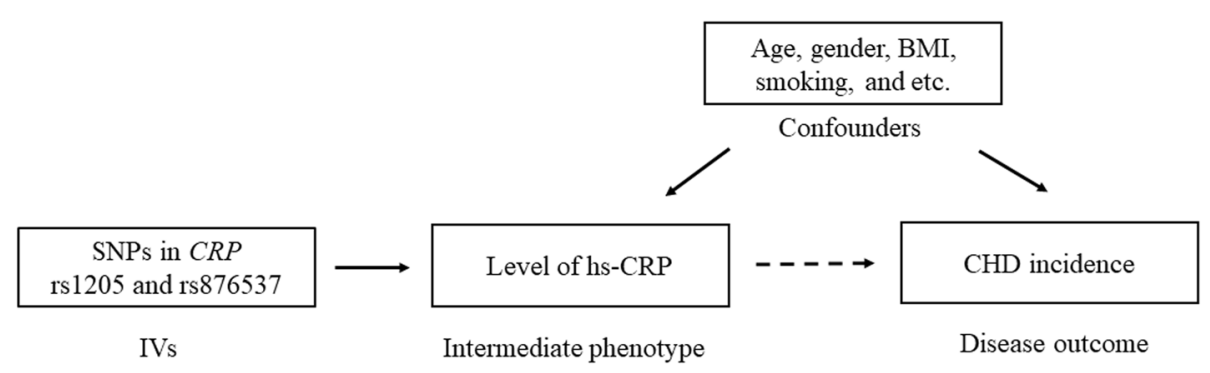

Fig. 1 The basic principle of Mendelian Randomization analysis for the current study. BMI, body mass index; CRP, C-reactive protein; CHD, coronary heart disease; IVs, instrumental variables; SNP, single nucleotide polymorphisms 
Table 1 Baseline characteristics for different hs-CRP groups

\begin{tabular}{|c|c|c|c|c|c|}
\hline \multirow[t]{2}{*}{ Variables } & \multirow[t]{2}{*}{ Group } & \multirow{2}{*}{$\begin{array}{l}\text { HRG } \\
n=1695\end{array}$} & \multirow{2}{*}{$\begin{array}{l}\text { NG } \\
n=2107\end{array}$} & \multirow[t]{2}{*}{$t / x^{2}$} & \multirow[b]{2}{*}{$P$} \\
\hline & & & & & \\
\hline Age & & $62.30 \pm 10.84$ & $58.62 \pm 10.34$ & 10.639 & $<0.001$ \\
\hline \multirow[t]{2}{*}{ Gender, n (\%) } & Male & $652(38.47)$ & $920(43.66)$ & 10.546 & $<0.001$ \\
\hline & Female & 1043(61.53) & $1187(56.34)$ & & \\
\hline \multirow[t]{2}{*}{ Drinking, n (\%) } & Yes & $346(20.41)$ & 499(23.68) & 5.811 & 0.016 \\
\hline & No & 1349(79.59) & 1608(76.32) & & \\
\hline \multirow[t]{2}{*}{ Smoking, n (\%) } & Yes & $383(22.59)$ & $556(26.39)$ & 7.264 & 0.007 \\
\hline & No & $1312(77.41)$ & $1551(73.61)$ & & \\
\hline BMI $\left(\mathrm{kg} / \mathrm{m}^{2}\right)$ & & $24.99 \pm 3.56$ & $23.56 \pm 3.11$ & 13.267 & $<0.001$ \\
\hline $\mathrm{SBP}(\mathrm{mmHg})$ & & $135.12 \pm 15.78$ & $132.18 \pm 15.37$ & 5.791 & $<0.001$ \\
\hline $\mathrm{DBP}(\mathrm{mmHg})$ & & $83.58 \pm 8.47$ & $83.09 \pm 8.58$ & 1.753 & 0.08 \\
\hline \multirow[t]{2}{*}{ Hypertension, n (\%) } & Yes & $914(53.92)$ & $918(43.57)$ & 40.336 & $<0.001$ \\
\hline & No & $781(46.08)$ & $1189(56.43)$ & & \\
\hline $\mathrm{TC}(\mathrm{mmol} / \mathrm{L})$ & & $4.96 \pm 1.06$ & $4.74 \pm 1.01$ & 6.407 & $<0.001$ \\
\hline $\mathrm{TG}(\mathrm{mmol} / \mathrm{L})$ & & $1.87 \pm 1.50$ & $1.53 \pm 1.31$ & 7.242 & $<0.001$ \\
\hline $\mathrm{HDL}-\mathrm{C}(\mathrm{mmol} / \mathrm{L})$ & & $1.35 \pm 0.32$ & $1.39 \pm 0.33$ & 3.705 & $<0.001$ \\
\hline $\mathrm{LDL}-\mathrm{C}(\mathrm{mmol} / \mathrm{L})$ & & $2.77 \pm 0.83$ & $2.67 \pm 0.77$ & 3.909 & $<0.001$ \\
\hline $\mathrm{GLU}(\mathrm{mmol} / \mathrm{L})$ & & $5.80 \pm 2.02$ & $5.49 \pm 1.62$ & 5.273 & $<0.001$ \\
\hline \multirow[t]{3}{*}{ T2DM, n (\%) } & T2DM & $222(13.10)$ & 184(8.73) & 35.962 & $<0.001$ \\
\hline & IFG & $418(24.66)$ & $426(20.22)$ & & \\
\hline & No & $1055(62.24)$ & 1497(71.05) & & \\
\hline
\end{tabular}

$B M I$ body mass index, DBP diastolic blood pressure, GLU glucose, $H D L-C$ high-density lipoprotein cholesterol, $H G R$ high risk group, IFG impaired fasting glucose, LDL-C low-density lipoprotein cholesterol, NG normal group, SBP systolic blood pressure, TC total cholesterol, TG triglyceride, T2DM Type 2 diabetes mellitus

Table 2 Association analysis of CRP polymorphisms with CHD incidence

\begin{tabular}{|c|c|c|c|c|c|c|c|}
\hline \multirow[t]{2}{*}{ SNP } & \multirow[t]{2}{*}{ Genotype } & \multirow[t]{2}{*}{$\mathrm{N}$} & \multirow{2}{*}{$\begin{array}{l}\text { Person- } \\
\text { years }\end{array}$} & \multirow{2}{*}{$\begin{array}{l}\text { Incidence } \\
\text { density } \\
\text { (/104 } \\
\text { Person- } \\
\text { years) }\end{array}$} & \multicolumn{3}{|l|}{$H R(95 \% C)^{a}$} \\
\hline & & & & & Additive model & Dominant model & Recessive model \\
\hline \multirow[t]{3}{*}{ rs10737175 } & CC & 56 & $12,085.13$ & 46.34 & $1.03(0.75-1.41)$ & $1.00(0.68-1.48)$ & $1.19(0.55-2.57)$ \\
\hline & $\mathrm{CT}$ & 35 & 7514.93 & 46.57 & $P=0.873$ & $P=0.995$ & $P=0.668$ \\
\hline & $\pi$ & 7 & 1246.34 & 56.16 & & & \\
\hline \multirow[t]{3}{*}{ rs1205 } & $\mathrm{CC}$ & 34 & 6872.61 & 49.47 & $1.03(0.78-1.36)$ & $0.90(0.60-1.35)$ & $1.26(0.79-2.03)$ \\
\hline & $\mathrm{CT}$ & 43 & $10,196.32$ & 42.17 & $P=0.851$ & $P=0.615$ & $P=0.338$ \\
\hline & $\pi$ & 21 & 3756.4 & 55.9 & & & \\
\hline \multirow[t]{3}{*}{ rs2808630 } & $\Pi$ & 71 & $14,371.91$ & 49.4 & $0.81(0.55-1.19)$ & $0.80(0.52-1.23)$ & $0.64(0.15-2.72)$ \\
\hline & $\mathrm{TC}$ & 24 & 5901.37 & 40.67 & $P=0.274$ & $P=0.300$ & $P=0.546$ \\
\hline & $\mathrm{CC}$ & 3 & 563.1 & 53.28 & & & \\
\hline \multirow[t]{3}{*}{ rs876537 } & CC & 34 & 6849.88 & 49.64 & $1.03(0.78-1.36)$ & $0.91(0.61-1.37)$ & 1.24(0.77-1.99) \\
\hline & $\mathrm{CT}$ & 43 & $10,189.35$ & 42.2 & $P=0.848$ & $P=0.659$ & $P=0.374$ \\
\hline & $\pi$ & 21 & 3802.17 & 55.23 & & & \\
\hline
\end{tabular}

Additive model: wild type vs heterozygote vs mutant Dominant model: wild type vs heterozygote + mutant Recessive model: wild type + heterozygote vs mutant $H R$ hazard ratio, $\mathrm{Cl}$ interval confidence

${ }^{a}$ Adjusted for age, gender, BMI, HDL-C, LDL-C, TC, TG, smoking, drinking, hypertension and T2DM 
Table 3 Association analysis of hs-CRP with CHD in the follow-up study

\begin{tabular}{|c|c|c|c|c|c|c|c|c|}
\hline Variables & Group & $\mathrm{N}$ & person-years & $\begin{array}{l}\text { Incidence density } \\
\left(/ 10^{4} \text { Person-years) }\right.\end{array}$ & $H R(95 \% C l)^{a}$ & $p^{a}$ & $H R(95 \% C l)^{b}$ & $p^{b}$ \\
\hline \multirow[t]{5}{*}{ hs-CRP } & & 98 & $20,836.52$ & 47.03 & $1.02(1.00-1.05)$ & 0.085 & $1.01(0.98-1.04)$ & 0.491 \\
\hline & $<1.0 \mathrm{mg} / \mathrm{L}$ & 37 & $10,951.48$ & 33.79 & $2.05(1.36-3.09)$ & 0.001 & $1.54(0.99-2.36)$ & 0.051 \\
\hline & $\geq 1.0 \mathrm{mg} / \mathrm{L}$ & 61 & 8582.37 & 71.08 & & & & \\
\hline & $<1.08 \mathrm{mg} / \mathrm{L}$ & 38 & $11,525.09$ & 32.97 & $2.23(1.48-3.35)$ & $<0.001$ & $1.69(1.11-2.60)$ & 0.016 \\
\hline & $\geq 1.08$ mg/L & 60 & 8008.77 & 74.92 & & & & \\
\hline
\end{tabular}

$H R$ hazard ratio, $\mathrm{Cl}$ interval confidence

${ }^{\text {a }}$ Crude model

${ }^{\mathrm{b}}$ Adjusted for age, gender, BMI, HDL-C, LDL-C, TC, TG, smoking, drinking, hypertension and T2DM

for age, gender, BMI, TC, TG, HDL-C, LDL-C, smoking, drinking, T2DM and hypertension, the adjust OR $(95 \%$ $C I)$ was $1.07(0.69-2.18)$ with a $P$ value of 0.777 .

\section{Discussion}

The present study firstly applied MR design to explore the casual effect of hs-CRP and CHD risk in Chinese Han population. The main results showed that CRP rs1205 and rs876537 were correlated with serum hsCRP level, but no causal effect of elevated hs-CRP on $\mathrm{CHD}$ risk was observed. Although the association between hs-CRP and CHD is unlikely to be causal, hs-CRP might be a predictor for incidence of CHD in general population.

Epidemiological studies have shown that plasma levels of hs-CRP are a strong independent predictor of risk of future CVDs [17, 18]. As a well-known inflammatory biomarker, hs-CRP is significantly elevated in patients dying suddenly with severe coronary artery diseases, both with and without acute coronary thrombosis [19]. There is considerable interest in establishing whether hs-CRP has a causal role in CHD. MR study, an IVbased method to infer the causality between intermediate phenotypes and disease, has been widely conducted in cardiovascular disease research [20]. Genetic variants that are associated with intermediate phenotypes are introduced as IVs in MR to estimate the effect of phenotypic exposures on disease outcome.

Table 4 The Mendelian randomization analysis of hs-CRP with $\mathrm{CHD}$

\begin{tabular}{lcclll}
\hline Methods & OR & \multicolumn{2}{c}{$95 \% \mathrm{Cl}$} & SE & $P$ \\
\cline { 3 - 4 } & & Lower & Upper & & \\
\hline IVW & 1.04 & 0.67 & 1.62 & 0.23 & $0.855^{\mathrm{a}}$ \\
& 1.07 & 0.69 & 2.18 & 0.22 & $0.777^{\mathrm{b}}$ \\
\hline
\end{tabular}

IVW inverse-variance weighted, $O R$ odds ratio, $C$ l confidence interval, SE Standard error

${ }^{a}$ Crude model

${ }^{\mathrm{b}}$ Adjusted for age, gender, BMI, HDL-C, LDL-C, TC, TG, smoking, drinking, hypertension and T2DM
Previous large sample size studies showed that no association of variants in the CRP locus and CHD were found, arguing against a causal role for CRP in atherosclerosis [21]. Another large-scale MR research selected CRP rs1205 as one of IVs and indicated that CRP was unlikely to be even a modest causal factor in CHD [13]. As a non-specific inflammatory biomarker of CRP, hsCRP takes the advantages of a higher sensitivity, accuracy and reproducibility. Our novel results firstly indicate that genetically raised hs-CRP are unrelated to the risk of CHD, which are consistent with previous studies [20, 21]. These findings imply that the cardiologists should treat CRP or hs-CRP as genuine confounders and pay more attention to other inflammatory markers in highrisk individuals.

Although American Centers for Disease Control and the American Heart Association has recommended hsCRP $1.0 \mathrm{mg} / \mathrm{L}$ as a cut-off value to identify individuals who is under a high risk of inflammatory reaction [16], no study has proved $1.0 \mathrm{mg} / \mathrm{L}$ is applicable to Chinese population. The SROC method was used to explore the hs-CRP cut-off value when evaluating the association of CHD. Our result showed hs-CRP $1.08 \mathrm{mg} / \mathrm{L}$ is the cutoff value with a relative high sensitivity and specificity to predict CHD incidence, inferring the criterion of $1.0 \mathrm{mg} /$ $\mathrm{L}$ is still suitable in China.

The current study investigated the cause effect of hsCRP and CHD risk in a prospective cohort study. The strengths of this study should be noted. First, for the expensive cost of hs-CRP measurement, limited MR studies have carried out to evaluate the association of hsCRP with CHD in a prospective cohort study. All the participants with a measurement of hs-CRP was enrolled in the follow-up study, the relative large sample size in our study substantially enhanced the statistical power, providing an objective and credible result on he-CRP and CHD. Second, it was firstly to explore the cut-off value of hs-CRP on predicting CHD incidence in Chinese Han population.

Several limitations were existed in the current study. First of all, the common SNPs that significantly 
correlated with hs-CRP are not derived from genomewide association study data. In addition, the causal effect of hs-CRP on sub-types of CHD were not investigated. Besides, the pleiotropy effect of SNPs in MR analysis was not evaluated. Last but not least, during acute events, the CRP gene expression can be up-regulated, leading to cascades that would increase the hs-CRP levels. However, our data might not accurately represent the fundamental of gene expression related to hs-CRP.

\section{Conclusion}

In conclusion, $C R P$ rs1205 and rs876537 variation were correlated with hs-CRP level.

Although the association between hs-CRP and CHD is unlikely to be causal, hs-CRP might be a predictor for incidence of $\mathrm{CHD}$ in general population.

\section{Supplementary information}

Supplementary information accompanies this paper at https://doi.org/10. 1186/s12881-019-0910-z

Additional file 1. Table S1. Bioinformatics Analysis of 4 selected SNPS in CRP Gene. Table S2. Stratification analysis of CRP gene polymorphisms and CHD. Table S3. Partial correlation analysis of CRP variation with hsCRP. Figure S1. Survival ROC analysis for hs-CRP and CHD incidence.

\begin{abstract}
Abbreviations
CHD: Coronary heart disease; Cl: Confidence interval; CVDs: Cardiovascular diseases; DL-C: High-density lipoprotein cholesterol; GLU: Glucose; HR: Hazard ratio; HRG: High risk group; Hs-CRP: High-sensitivity C-reactive protein IV: Instrumental variable; LDL-C: Low-density lipoprotein cholesterol; MAF: Minor allele frequency; MR: Mendelian Randomization; NG: Normal group; OR: Odds Ratios; PCR: Polymerase chain reaction; SD: Standard deviation; SNP: Single nucleotide polymorphism; SROC: Survival receiver operator characteristic curve; T2DM: Type 2 diabetes mellitus; TC: Total cholesterol; TG: Triglycerides
\end{abstract}

\section{Acknowledgments}

First and foremost, I would like to show my deepest gratitude to Dr. Song Yang and Prof. Chong Shen, who are respectable, responsible and resourceful scholars, providing me with valuable guidance in every stage of the writing of this thesis. Their keen and vigorous academic observation enlightens me not only in this thesis but also in my future study. I shall extend my thanks to Mr. Shen for all his kindness and help. My sincere appreciation also goes to my colleagues from Department of Cardiology, Affiliated Yixing People's Hospital of Jiangsu University, who participated this study with great cooperation.

\section{Authors' contributions}

SY and CS conceived and designed the study. Clinical data was collected and collated by QZ, YC, XZ, JS, PW. XC and YJ analyzed the data. QZ wrote the paper. SY and CS reviewed and edited the manuscript. All authors read and approved the manuscript.

\section{Funding}

This work was supported by the National Natural Science Foundation of China (Grant No.81573232), The funders had no role in study design, data collection and analysis, decision to publish, or preparation of the manuscript.

\section{Availability of data and materials}

The datasets used and/or analyzed during the current study are available from the corresponding author on reasonable request.

\section{Ethics approval and consent to participate}

Written informed consent was obtained from all participants. The research protocol was approved by the ethics committee of Nanjing Medical University and written informed consent was obtained from all subjects during epidemiological interviews.

\section{Consent for publication}

Not applicable.

\section{Competing interests}

The authors declare that they have no competing interests.

\section{Author details}

'Department of Cardiology, Affiliated Yixing People's Hospital of Jiangsu University, Yixing, China. ${ }^{2}$ Department of Epidemiology, School of Public Health, Nanjing Medical University, Nanjing, China. ${ }^{3}$ Department of Clinical Epidemiology, Children's Hospital of Fudan University, Shanghai 201102, China.

Received: 17 April 2019 Accepted: 23 October 2019

Published online: 06 November 2019

\section{References}

1. He J, et al. Major causes of death among men and women in China. N Engl J Med. 2005:353(11):1124-34

2. Ripatti S, et al. A multilocus genetic risk score for coronary heart disease: case-control and prospective cohort analyses. Lancet. 2010; 376(9750):1393-400.

3. Ross R. Atherosclerosis-an inflammatory disease. N Engl J Med. 1999;340(2):115-26.

4. Buckley DI, et al. C-reactive protein as a risk factor for coronary heart disease: a systematic review and meta-analyses for the U.S. preventive services task force. Ann Intern Med. 2009;151(7):483-95.

5. Mirhafez SR, et al. Serum high-sensitivity C-reactive protein as a biomarker in patients with metabolic syndrome: evidence-based study with 7284 subjects. Eur J Clin Nutr. 2016;70(11):1298-304.

6. Sugiyama T, et al. Relationship between serum high-sensitivity C-reactive protein and myocardial infarction in a general Japanese population. J Clin Lab Anal. 2016;30(6):999-1002.

7. Ridker PM, et al. Rosuvastatin to prevent vascular events in men and women with elevated C-reactive protein. N Engl J Med. 2008;359(21):2195-207.

8. Pepys MB. C-reactive protein is neither a marker nor a mediator of atherosclerosis. Nat Clin Pract Nephrol. 2008;4(5):234-5.

9. Zacho J, et al. Genetically elevated C-reactive protein and ischemic vascular disease. N Engl J Med. 2008;359(18):1897-908.

10. Bowden J, Davey Smith G, Burgess S. Mendelian randomization with invalid instruments: effect estimation and bias detection through egger regression. Int J Epidemiol. 2015;44(2):512-25.

11. Smith GD, Ebrahim S. 'Mendelian randomization': can genetic epidemiology contribute to understanding environmental determinants of disease? Int J Epidemiol. 2003;32(1):1-22.

12. Hartwig FP, et al. Two-sample Mendelian randomization: avoiding the downsides of a powerful, widely applicable but potentially fallible technique. Int J Epidemiol. 2016;45(6):1717-26.

13. Collaboration, C.R.P.C.H.D.G, et al. Association between $C$ reactive protein and coronary heart disease: mendelian randomisation analysis based on individual participant data. BMJ. 2011;342:d548.

14. Swerdlow DI, et al. HMG-coenzyme a reductase inhibition, type 2 diabetes, and bodyweight: evidence from genetic analysis and randomised trials. Lancet. 2015:385(9965):351-61.

15. Chen J, et al. Common variants in TGFBR2 and miR-518 genes are associated with hypertension in the Chinese population. Am J Hypertens. 2014;27(10):1268-76.

16. Ridker PM. Inflammation in atherothrombosis: how to use high-sensitivity C-reactive protein (hsCRP) in clinical practice. Am Heart Hosp J. 2004;2(4 Suppl 1):4-9.

17. Ridker PM, et al. C-reactive protein and other markers of inflammation in the prediction of cardiovascular disease in women. N Engl J Med. 2000; 342(12):836-43.

18. Tracy RP, et al. Relationship of C-reactive protein to risk of cardiovascular disease in the elderly. Results from the cardiovascular health study and the rural health promotion project. Arterioscler Thromb Vasc Biol. 1997;17(6):1121-7. 
19. Burke AP, et al. Elevated C-reactive protein values and atherosclerosis in sudden coronary death: association with different pathologies. Circulation. 2002;105(17):2019-23.

20. Kawashiri MA, et al. Mendelian randomization: its impact on cardiovascular disease. J Cardiol. 2018;72(4):307-13.

21. Elliott $\mathrm{P}$, et al. Genetic loci associated with $\mathrm{C}$-reactive protein levels and risk of coronary heart disease. JAMA. 2009:302(1):37-48.

\section{Publisher's Note}

Springer Nature remains neutral with regard to jurisdictional claims in published maps and institutional affiliations.

Ready to submit your research? Choose BMC and benefit from:

- fast, convenient online submission

- thorough peer review by experienced researchers in your field

- rapid publication on acceptance

- support for research data, including large and complex data types

- gold Open Access which fosters wider collaboration and increased citations

- maximum visibility for your research: over $100 \mathrm{M}$ website views per year

At BMC, research is always in progress.

Learn more biomedcentral.com/submissions 